\title{
Effects of lentiviral infection of mesenchymal stem cells on the expression of octamer transcription factor 4
}

\author{
JING CHANG, LI TANG, HAN LEI, XIAO-GANG ZHANG, ZHONG ZUO, WEI HUANG and HANG FU
}

\begin{abstract}
Department of Cardiology, The First Affiliated Hospital of Chongqing Medical University, Chongqing 400016, P.R. China
\end{abstract}
Received September 4, 2013; Accepted April 16, 2014

DOI: $10.3892 / \mathrm{mmr} .2014 .2505$

\begin{abstract}
The present study aimed to investigate the effects of lentiviral infection of human umbilical cord mesenchymal stem cells (hUCMSCs) on the expression of octamer transcription factor 4 (Oct4). hUCMSCs were infected with lentivirus carrying the green fluorescent protein gene $(G F P)$ at different multiplicities of infection (MOI), and the optimal MOI was determined by flow cytometry; the proliferation of non-infected and GFP-carrying lentivirus-infected hUCMSCs was evaluated by the MTT assay; and the expression of the Oct4 gene was measured by quantitative reverse transcription-polymerase chain reaction (qRT-PCR) and immunofluorescence staining in hUCMSCs cultured in vitro for eight weeks. Positive GFP staining of hUCMSCs was estimated at $>75 \%$ at $48 \mathrm{~h}$ following infection with the GFP-carrying lentivirus (MOI $=20$ ); no effect on hUCMSC proliferation was detected by the MTT assay following the infection; immunofluorescence analysis detected positive Oct4 expression in the cell nuclei at two and eight weeks of culture, while the relative expression of Oct4 assessed by qRT-PCR was $0.9075 \pm 0.0124$. The GFP gene carried by the lentivirus was successfully expressed in hUCMSCs and had no significant effect on Oct4 expression, which lays a solid foundation for future studies investigating gene functions via the use of exogenous markers.
\end{abstract}

\section{Introduction}

Stem cell research is an emerging and actively studied topic in life sciences. Despite the pluripotency of embryonic stem cells (ESCs), their use is currently limited, largely due to ethical issues. Since 2006, transformation of fibroblasts into pluripotent cells has been successfully induced by introduction of four genes: Oct4, Sox2, c-Myc and Klf4. The resulting

Correspondence to: Dr Jing Chang, Department of Cardiology, The First Affiliated Hospital of Chongqing Medical University, No. 1 Yuanjiagang Youyi Road, Chongqing 400016, P.R. China E-mail: jingchangcn@126.com

Key words: green fluorescent protein, lentivirus, human umbilical cord mesenchymal stem cells, octamer transcription factor 4 induced pluripotent stem (iPS) cells, have a high similarity with ESCs in morphology, proliferation, surface marker and gene expression profiles, and differentiation potential $(1,2)$. Since then, a novel method to efficiently induce transformation of skin cells into iPS cells was also identified, involving introduction of the microRNA (mir) that is regulated by the the Oct4 and Sox 2 transcription factors, miR-302 (3-5).

Human umbilical cord mesenchymal stem cells (hUCMSC), are a type of adult stem cells with multidirectional differentiation potential that can differentiate into cardiomyocytes (6), vascular endothelial cells and osteoblasts (7), etc.; this type of mesenchymal stem cells (MSCs) is widely used in research and clinical applications.

The octamer transcription factor 4 (Oct4), also known as Oct $3 / 4$ or Pou $5 f l$ (POU domain, class 5 , transcription factor 1) is a homeodomain transcription factor of the POU family, and the most important transcriptional regulator of self-renewal and differentiation in ESCs. Oct4 is considered a key factor of the stem cellmultidirectional differentiation potential (8).

In this study, we infected hUCMSCs with a lentivirus that carries the green fluorescent protein gene $(G F P)$ to assess its effects on Oct4 expression and explore the optimal conditions for future transfections of hUCMSCs with the GFP-conjugated exogenous mir-302.

\section{Materials and methods}

hUCMSC isolation and cultures. Five centimeters of the umbilical cord were collected from full-term newborns delivered by caesarean section. Informed consent of the mother, who faced no complications throughout pregnancy was obtained. The umbilical cord was immersed in a sterile culture flask containing Dulbecco's modified Eagle's medium (DMEM)/F12 (Gibco-BRL, Grand Island, NY, USA) and was placed on a clean bench; the arteries, veins and Wharton's jelly were removed from the umbilical cord, followed by washing with normal saline to remove residual blood. Next, the umbilical cord was cut into $1-\mathrm{mm}^{3}$ pieces and seeded in a culture flask within a small volume of DMEM/F12 medium containing $10 \%$ fetal bovine serum (FBS). The flask was placed in a $37^{\circ} \mathrm{C}$ incubator for $30 \mathrm{~min}$; then, part of the medium was added into wet tissue pieces, which were incubated in a humidified $37^{\circ} \mathrm{C}$, $5 \% \mathrm{CO}_{2}$ incubator. Half of the culture medium was replaced three days later and cell growth was observed daily under an inverted microscope. 
Lentivirus packaging. A lentivirus packaging system, including pLenO-DCE-Puro, pRSV-Rev, pMDlg-pRRE and pMD2.G was purchased from (Invitrogen, Carlsbad, CA, USA). HEK-293T cells (Invitrogen) were cultured until $60-70 \%$ confluence was reached, then washed with PBS twice, isolated by treating with $0.25 \%$ trypsin containing $0.01 \%$ EDTA (Sigma, St. Louis,USA) for 6-7 minutes. Cells were counted with a hemocytometer and $2 \times 10^{6}$ were seeded into $10-\mathrm{cm}^{2}$ petri dishes and incubated overnight in a $37^{\circ} \mathrm{C}$ incubator with $5 \% \mathrm{CO}_{2}$. The medium was changed when the cells had reached $60-70 \%$ confluence. The $10 \mu \mathrm{l}$ plasmid (pRSV-Rev, pMFlg-pRRE and pMD2.G; Invitrogen) and $3 \mu \mathrm{l}$ calcium phosphate (Sigma, St. Louis, MO, USA) mixture was transferred in medium containing monolayer cells and was gently mixed; the medium was changed after $6 \mathrm{~h}$ of culture. The supernatant containing the virus was collected $48 \mathrm{~h}$ following the infection and centrifuged at 1,000 $\mathrm{x}$ g for $10 \mathrm{~min}$ at $4^{\circ} \mathrm{C}$; the supernatant was passed through a $0.45-\mu \mathrm{m}$ filter. Following a new centrifugation at $6,000 \mathrm{x}$ g for $2 \mathrm{~h}$ at $4^{\circ} \mathrm{C}$, the pellet containing the virus was dissolved in serum-free culture medium, aliquoted and stored at $-70^{\circ} \mathrm{C}$ prior to further use.

Lentivirus titer determination. One day before measurement, HEK-293T cells were seeded into 96-well plates with $100 \mu \mathrm{l}$ of culture medium in each well. The lentivirus was serially diluted over the wells of the plate; $8 \mu \mathrm{f} / \mu \mathrm{l}$ polybrene (Sigma) was simulteneously added to increase the efficiency of infection. Cell growth was observed two days later and the cells were collected for subsequent titer determination.

Measurement of infection efficiency. hUCMSCs at passage 3 were isolated as described, seeded into 24-well plates $\left(5 \times 10^{4}\right.$ cells/well) containing DMEM/F12 medium supplemented with $10 \% \mathrm{FBS}$, and incubated in a $37^{\circ} \mathrm{C}$ incubator with $5 \% \mathrm{CO}_{2}$. Lentivirus [multiplicity of infection $(\mathrm{MOI})=0,5,10,15,20$; triplicates for each MOI value] was added to the wells when the cells had reached $70-80 \%$ confluence. The medium was changed $24 \mathrm{~h}$ following the infection and the fluorescence intensity was measured after 24, 48, 72 and $96 \mathrm{~h}$. Non-infected hUCMSCs were used as negative controls. The two cell groups were respectively harvested by $0.25 \%$ trypsin (Hyclone, Logan, UT, USA), digested for $2 \mathrm{~min}$ at room temperature and resuspended in phosphate-buffered saline (PBS) after $96 \mathrm{~h}$ of infection. The efficiency of infection was shown by the percentage of cells containing GFP and was calculated by the following formula: (Number of GFP positive cells/total number of cells) x 100 . The cell number was determined by a BD FACSCanto II cytometer (BD Biosciences, San José, CA, USA).

MTT assay. hUCMSCs at passage 3 were isolated as described and seeded into 96 -well plates $\left(2 \times 10^{3}\right.$ cells/well). Five wells of the non-infected and the lentivirus-infected group were used for each assay, containing PBS and an equal volume of lentivirus at a MOI of 20, respectively. At 24, 48, 72 and $96 \mathrm{~h}$ after the infection, $20 \mu \mathrm{l}$ of MTT (Beyotime Institute of Biotechnology, Haimen, China) were added to each well. Following a 4-h incubation in a $37^{\circ} \mathrm{C}$ incubator with $5 \% \mathrm{CO}_{2}$, the culture medium was removed and $100 \mu$ l of dimethyl sulfoxide were added to dissolve the formed crystals under low-speed vibration for $10 \mathrm{~min}$. The optical density (OD) was measured at $490 \mathrm{~nm}$ with a microplate reader 680 (Bio-Rad, Hercules, CA, USA).

Quantitative reverse transcription-polymerase chain reaction ( $q R T P C R)$. Total RNA was extracted from two- and eight-week cultures of hUCMSCs with the TRIzol reagent (Invitrogen, New York, NY, USA) following the manufacturer's instructions. The RNA was reverse transcribed into complementary DNA (cDNA) following instructions of the Takara PrimeScript TM RT-RCR kit (DRR014A; Takara Bio, Inc., Dalian, China). The forward and reverse primers were: 5'-GTGAGAG GCAACCTGGAGAAT-3; 5'-TACAGAACCACACTCGGAC CAC-3' for the gene Oct4 (Abcam, Cambridge, UK), and 5'-CTTTGGTATCGTGGAAGGACTC-3'; 5'-GTAGAGGCA GGGATGATGTTCT-3' for the glyceraldehyde 3-phosphate dehydrogenase gene $(G A P D H)$, respectively. The expected length of the amplified fragments was 118 and $132 \mathrm{bp}$, respectively. The qPCR reaction was performed with the SYBR Premix Ex Taq ${ }^{\mathrm{TM}}$ II kit (Bio-Rad, Hercules, CA, USA) using the following conditions: denaturation at $95^{\circ} \mathrm{C}$ for $30 \mathrm{sec}$, followed by 40 cycles of denaturation at $95^{\circ} \mathrm{C}$ for $5 \mathrm{sec}$, annealing at $55^{\circ} \mathrm{C}$ for $30 \mathrm{sec}$ and elongation at $72^{\circ} \mathrm{C}$ for $30 \mathrm{sec}$. Analysis of the amplification and melting curves was performed after the reaction. The expression of Oct4 was calculated by the $\Delta(\Delta \mathrm{Ct})$ method (9) and was expressed relative to that of GAPDH.

Immunofluorescence. When cells in 24-well plates had reached $65-70 \%$ confluence, the culture medium was discarded. The plates were washed twice in PBS, fixation with $4 \%$ formaldehyde at room temperature for $20 \mathrm{~min}$, three washes in PBS for 5 min, treatment with $0.2 \%$ Triton $\mathrm{X}-100$ for $30 \mathrm{~min}$, and three washes in PBS for 5 min. Next, blocking was performed in $1 \%$ bovine serum albumin for $1 \mathrm{~h}$, and the blocking buffer was washed away prior to the incubation with the 100X-diluted rabbit anti-human/mouse Oct4 antibody (ab18976, Abcam) at $4^{\circ} \mathrm{C}$ in a humid box overnight; PBS instead of the primary antibody was used as the blank control. The plates were placed at $37^{\circ} \mathrm{C}$ for $30 \mathrm{~min}$, followed by three PBS washes for 5 min, incubation with the 50x-diluted tetramethylrhodamine (TRITC)-labeled goat anti-rabbit secondary antibody IgG (Golden Bridge Biotechnology Co., Ltd., Beijing, China) in the dark at $37^{\circ} \mathrm{C}$ for $2 \mathrm{~h}$, three PBS washes for $5 \mathrm{~min}$, 4',6-diamidino-2-phenylindole (DAPI) nuclear staining for $1 \mathrm{~min}$ and a final PBS wash for $5 \mathrm{~min}$. Glass slides were mounted with glycerol and observed under a fluorescence microscope (Olympus, Tokyo, Japan).

Statistical analysis. Statistical analysis was performed with the SPSS 17.0 software (IBM, Armonk, NY, USA). Quantitative data were presented as mean \pm standard deviation $(\bar{x} \pm S D)$, and comparisons between groups was performed with t-tests. $\mathrm{P}<0.05$ was considered to indicate significant differences.

\section{Results}

hUCMSC growth and morphology. Half of the culture medium was replaced on the third day of the culture, when most of the tissue pieces had attached to the wells, and was again changed 

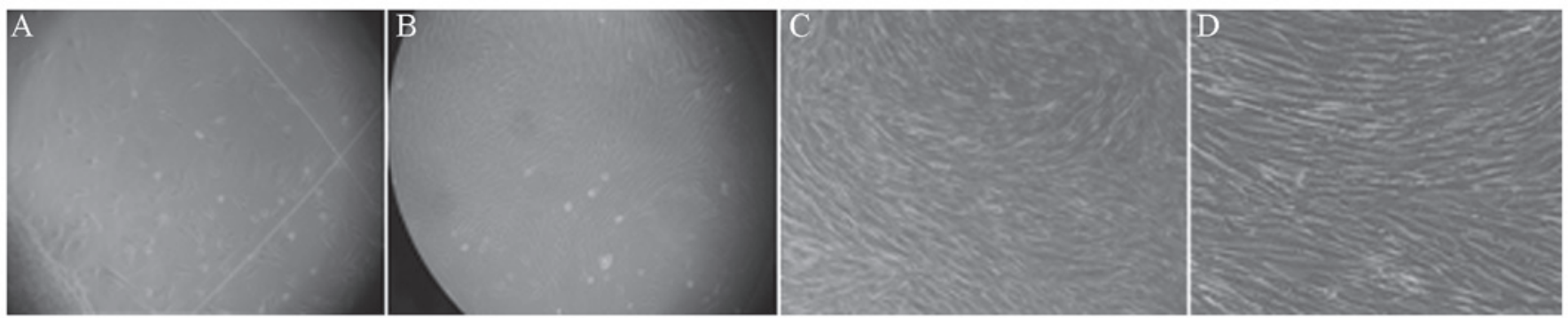

Figure 1. Morphology of human umbilical cord mesenchymal stem cells (hUCMSCs). (A and B) Primary culture at (A) 10 and (B) 15 days of culture; (C and D) Cells at passage 3 (C, x100; D, x200).
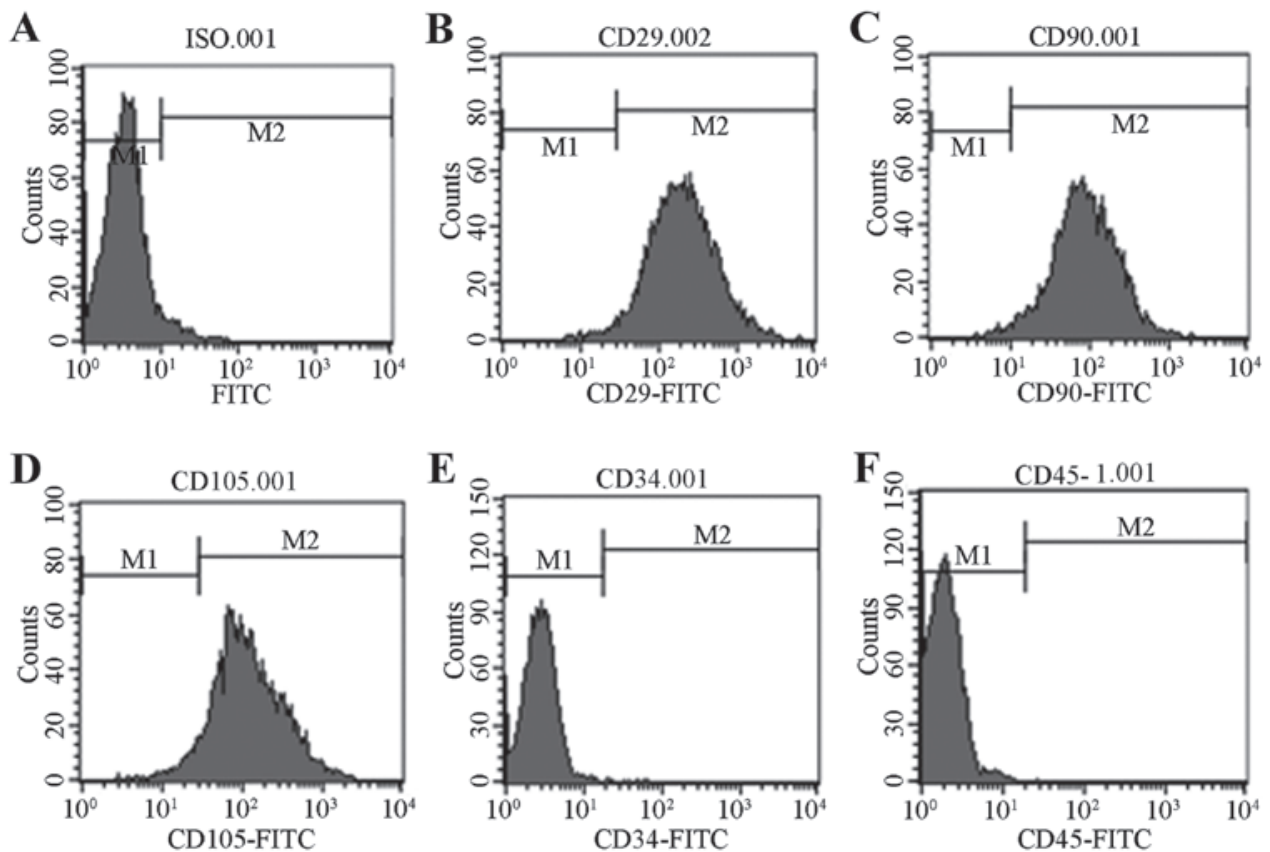

Figure 2. Expression of surface markers in human umbilical cord mesenchymal stem cells (hUCMSCs). hUCMSCs are positive for CD29, CD90 and CD105, and negative for CD34 and CD45.
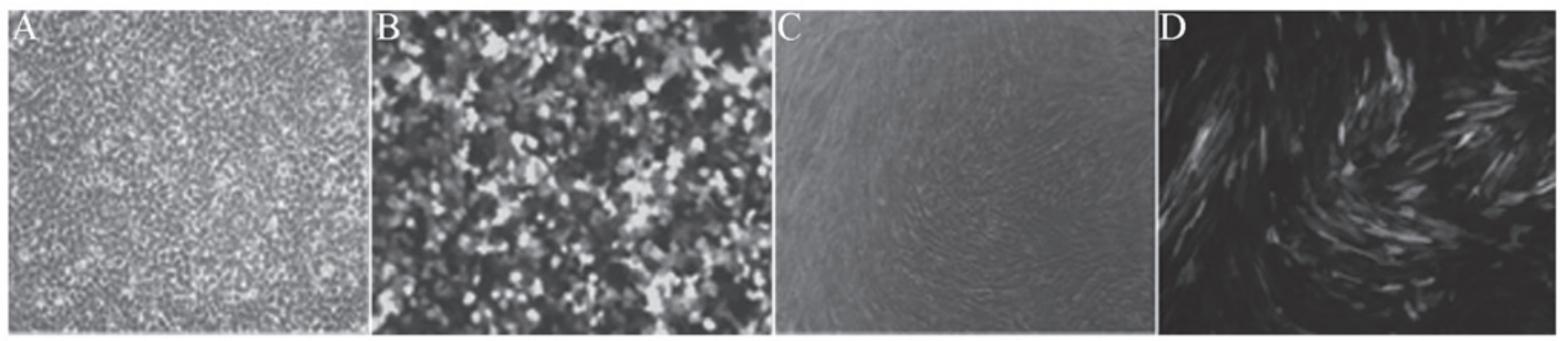

Figure 3. Green fluorescent protein (GFP) expression in HEK-293T cells and in human umbilical cord mesenchymal stem cells (hUCMSCs) following infection. (A and B) HEK-293T cells $48 \mathrm{~h}$ following the infection. (A) Light field (x100); (B) fluorescence field (x100). (C and D) hUCMSCs following the infection [multiplicity of infection (MOI) $=20,96 \mathrm{~h}$ ]. (C) Light field (x100); (D) fluorescence field (x100).

every two days afterwards. A few fusiform hUCMSCs dissociated from the tissue (Fig. 1A) after 10 days of culture, while a high number of colonies was formed after 15 days, when hUCMSCs displayed a typical fibroblast-like spindle shape (Fig. 1B). Cells were trypsinized and were passaged after they had reached $80-90 \%$ confluence. Cell morphology and properties, such as cell cycle phase (the majority were in G0/G1 phases with only a small minority in the S phase), self-renewal, pluripotency and differentiation did not significantly change after passage 10 (Fig. 1C and D).

Analysis of hUCMSC surface markers. The analysis of hUCMSCs at the third passage by flow cytometry showed that CD29, D90 and CD105 are expressed in these 

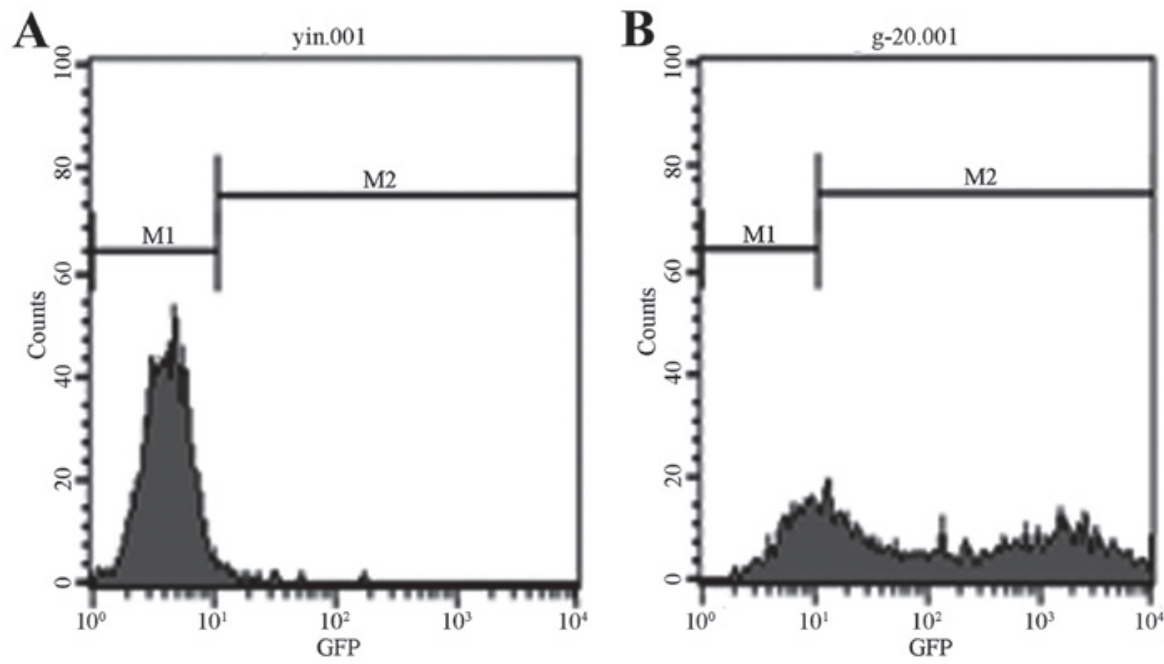

Figure 4. Analysis of the infection efficiency by flow cytometry. (A) Non-infected group; (B) lentivirus-infected group. The infection efficiency of the lentivirus-infected group [ $96 \mathrm{~h}$, multiplicity of infection $(\mathrm{MOI})=20]$ is markedly higher $(75.82 \%)$ compared to the non-infected group $(0.75 \%)$.

cells (Fig. 2A-C and E), while the cells are negative for CD34 and CD45 (Fig. 2F and G), which are the surface markers of hematopoietic stem cells.

Lentivirus packaging and infection efficiency. Bright green fluorescence was observed under the fluorescence microscope $48 \mathrm{~h}$ following infection of the HEK-293T cells (Fig. 3A and B), which indicated successful packaging of the active lentivirus. Hole-by-dilution was used to determine the virus titer and flow cytometry was employed to detect the percentage of GFP-positive cells. The titer of the GFP-carrying lentivirus was estimated at $2 \times 10^{8} \mathrm{TU} / \mathrm{ml}$, which was considered suitable for infecting hUCMSCs.

Following hUCMSC infection, all cells expressed the GFP protein as shown by green fluorescence emitted at any MOI value (except MOI $=0$ ). The cell morphology observed under the fluorescence microscope was similar to that observed under a light microscope (Fig. 3C and D).

The fluorescence intensity was the strongest at $96 \mathrm{~h}$ following the infection. With the increase of MOI and the proliferation of cells, fluorescence became stronger, reaching its highest level at MOI $=20$. Flow cytometry analysis showed that the infection efficiency is $75.85 \%$ at MOI $=20$ and at $96 \mathrm{~h}$ following the infection (Fig. 4).

Cell proliferation. Cell proliferation was measured in the non-infected and the lentivirus-infected group with the MTT assay (Fig. 5), and no statistically significant difference was observed between the OD values of the two groups $(\mathrm{P}>0.05)$, which suggested that the GFP gene carried by the lentivirus does not markedly affect cell proliferation under the tested conditions.

mRNA level of Oct4. The Oct4 expression level was defined as 1 in the lentivirus-infected group of hUCMSCs after two weeks of culture, and thus, the level of Oct4 was estimated at $0.9075 \pm 0.0124$ after eight weeks of culture (Fig. 6). The relative expression of $O c t 4$ was not significantly different between cells cultured for two and eight weeks $(\mathrm{P}>0.05)$.

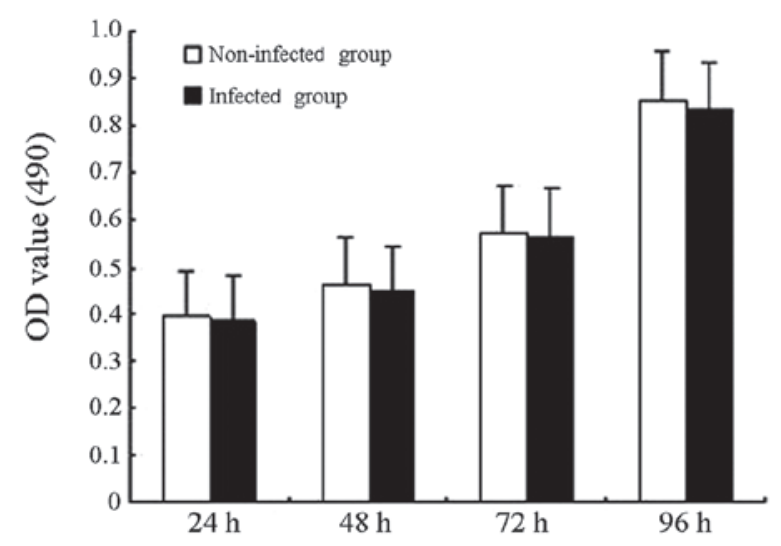

Figure 5. Proliferation of cells from the infected and the non-infected group, as assessed by the MTT assay. There is no statistically significant difference in the optical density (OD) values at $490 \mathrm{~nm}$ between the two groups.

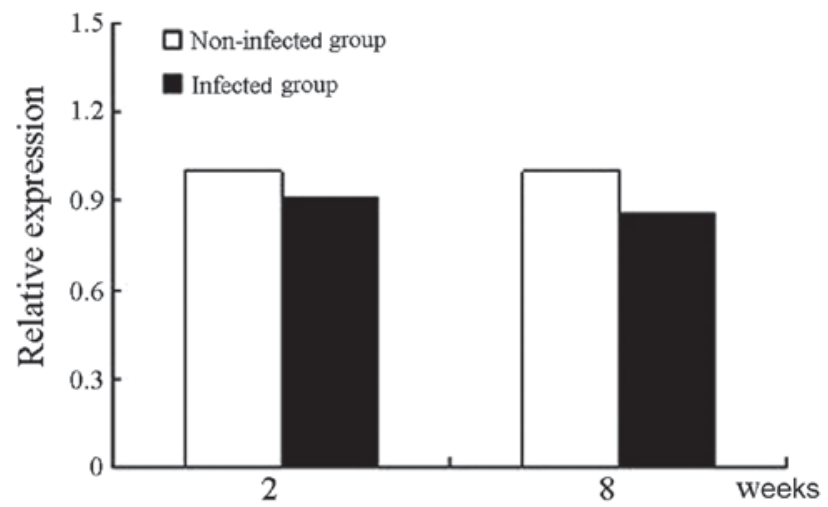

Figure 6. Expression of the octamer transcription factor 4 gene $($ Oct 4$)$ as assessed by quantitative reverse transcription-polymerase chain reaction (qRT-PCR). There is no statistically significant difference in the relative expression level of Oct4 between two and eight weeks of culture.

Oct4 protein expression. Immunofluorescence revealed that the Oct4 protein is expressed in hUCMSCs of both the infected and the non-infected group. There was no statistically 

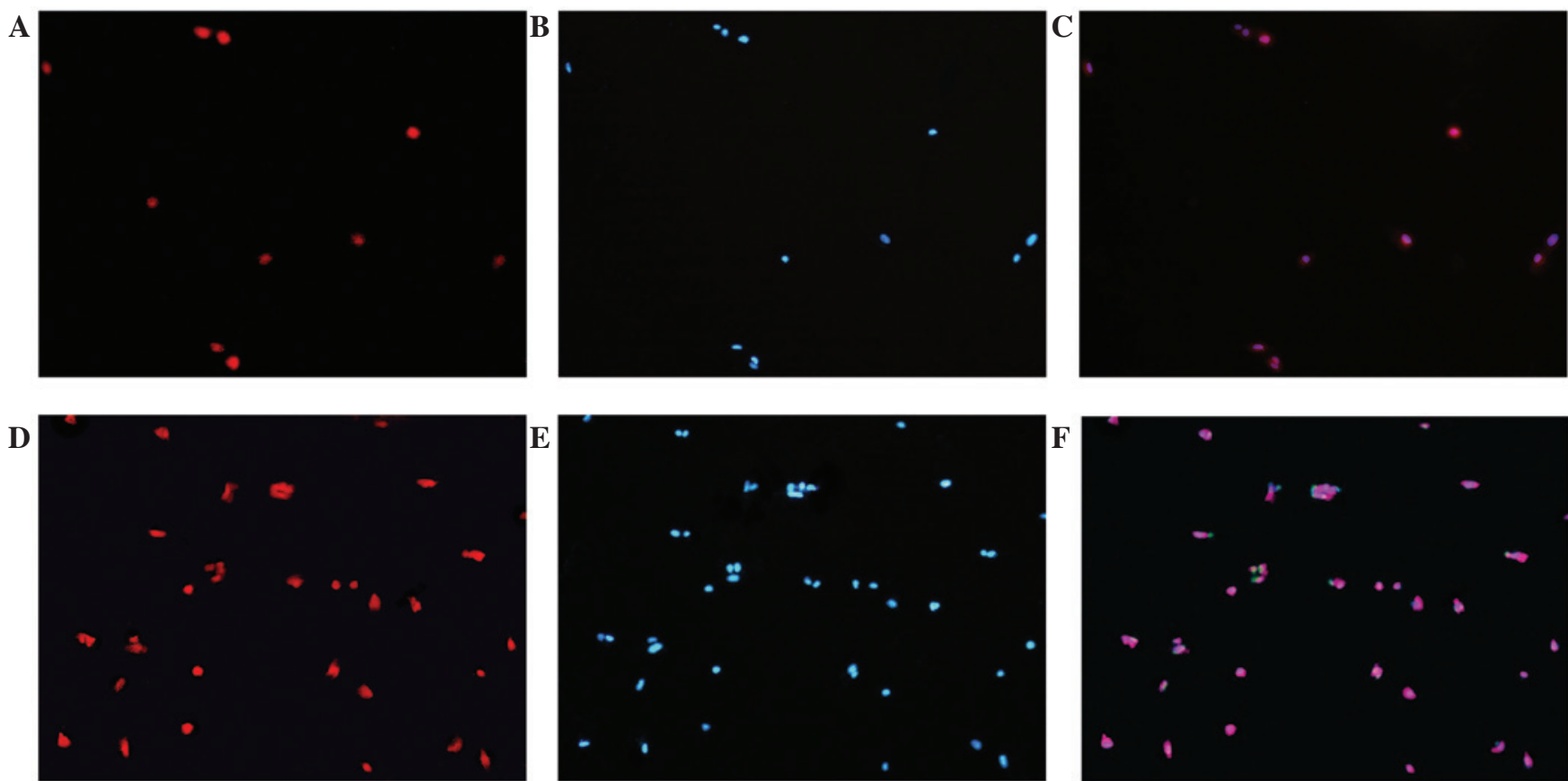

Figure 7. Octamer transcription factor 4 (Oct4) expression in human umbilical cord mesenchymal stem cells (hUCMSCs) observed under a fluorescence microscope (x100). (A-C) Non-infected group; (D-F) infected group. Immunofluorescence shows that the Oct4 protein is expressed in both the infected and the non-infected group. 4',6-diamidino-2-phenylindole (DAPI) staining shows that Oct4 mainly localizes in the cell nuclei.

significant difference in the levels of Oct4 between the two groups. DAPI nuclear staining experiments revealed that the Oct4 protein is mainly distributed in the cell nuclei (Fig. 7).

\section{Discussion}

MSCs, an important member of the stem cell family, are multipotent stem cells derived from the mesoderm at the early stages of development, and show high self-renewal ability and multidirectional differentiation potential. HUCMSCs, a type of adult stem cells with multidirectional differentiation potential (10), have several advantages compared to other MSCs, including easy access and availability, high proliferation ability, low immunogenicity, and no associated ethical limitations. Moreover, hUCMSCs remain in their primitive and undifferentiated state (11-14), which renders introduction and expression of exogenous genes easy during cell proliferation; this is one reason why hUCMSCs have become the focus of numerous studies in recent years. In our experiment, we cultured umbilical cord tissue sections in order to isolate hUCMSCs.

Oct4 has been recognized as a specific marker of stem cells and a key factor for cell totipotency, which is lost during cell differentiation (15). Oct4 is not only expressed in pluripotent embryonic cells, but also in adult stem cells (16-19). Carlin et al (20) demonstrated that the embryonic stem cell markers Oct4, Sox 2 and Nanog are expressed in hUCMSCs. Can et al (21) showed that the Oct4 gene is expressed in hUCMSCs, indicating that hUCMSCs possess stem cell properties.

Lentivirus is a non-oncogenic virus; in contrast to other viral vectors, it can infect dividing and non-dividing cells, especially cells that are difficult to transfect, such as primary cells, stem cells and neurons, with an infection efficiency of almost $100 \%$. In addition, lentiviral vectors can effectively integrate, and thus allow consistent expression of, exogenous genes into the chromosome of host cells $(22,23)$, and have attracted increasing attention in the field of gene transfer vectors (24). Miyoshi et al (25) demonstrated that GFP is a marker of infection efficiency. The lentiviral vector used in our experiment bears the GFP-encoding gene, in order to allow assessment of cell infection efficiency and optimization of the infection conditions. Lentiviral vectors may integrate close to promoters and insertion mutations, which may explain why infection with the GFP-carrying lentivirus does not affect hUCMSC proliferation. The low probability of lentivirus insertion near promoters, minimizes the occurrence of insertion mutations, which may partly explain the fact that proliferation of the infected cells was not changed.

In our experiments, the GFP gene was successfully introduced into hUCMSCs through a lentiviral vector, which provides an ideal model for subsequent research on gene infection. Green fluorescence was observed under a fluorescence microscope and was the strongest at $96 \mathrm{~h}$ post-infection; the cell morphology observed under the fluorescence microscope was similar to that observed under a light microscope. Flow cytometry showed that the infection efficiency was $>75 \%$ at MOI $=20$. The OD values from the MTT assay did not show any significant difference between the infected and the non-infected groups, which indicates that infection with a GFP-carrying lentivirus has no effect on cell proliferation. Oct4 expression was detected by qRT-PCR and immunofluorescence. qRT-PCR revealed that the Oct 4 mRNA level is not significantly different between cells cultured for two and eight weeks, which implies that infection of hUCMSCs with the GFP-carrying lentivirus does not affect 
their pluripotency. Immunofluorescence further showed that the Oct4 protein is expressed in both infected and non-infected cells, with no apparent difference between the two groups, and is mainly expressed in the cell nuclei.

In conclusion, the GFP-carrying lentivirus can effectively infect hUCMSCs and has no prominent effect on cell pluripotency and proliferation. Our results lay a solid foundation for future research using exogenous gene-carrying vectors.

\section{References}

1. Takahashi K and Yamanaka S: Induction of pluripotent stem cells from mouse embryonic and adult fibroblast cultures by defined factors. Cell 126: 663-676, 2006.

2. Okita K, Ichisaka $\mathrm{T}$ and Yamanaka S: Generation of germline-competent induced pluripotent stem cells. Nature 448: 313-317, 2007.

3. Lin SL, Chang DC, Lin CH, Ying SY, Leu D and Wu DT: Regulation of somatic cell reprogramming through inducible mir-302 expression. Nucleic Acids Res 39: 1054-1065, 2011.

4. Card DA, Hebbar PB, Li L, et al: Oct4/Sox2-regulated mir-302 targets cyclin D1 in human embryonic stem cells. Mol Cell Biol 28: 6426-6438, 2008.

5. Zovoilis A,Pantazi A, Smoraq L, et al: Embryonic stem cell-related miRNAs are involved in differentiation of pluripotent cells originating from the germ line. Mol Hum Reprod 16: 793-803, 2010.

6. Schmidt D, Mol A, Odermatt B, et al: Engineering of biologically active living heart valve leaflets using human umbilica cord-derived progenitor cells. Tissue Eng 12: 3223-3232, 2006.

7. Sarugaser R, Lickorish D, Baksh D, Hosseini MM and Davies JE: Human umbilical cord perivascular (HUCPV) cells: A source of mesenchymal progenitors. Stem Cells 23: 220-229, 2005.

8. Boyer LA, Lee TI, Cole MF, et al: Core transcriptional regulatory circuitry in human embryonic stem cells. Cell 122: 947-956, 2005.

9. Livak KJ and Schmittgen TD: Analysis of relative gene expression data using real-time quantitative PCR and the 2(-Delta Delta C(T)) method. Methods 25, 402-408, 2001.

10. Wang H, Yang Y, Ho G, et al: Programming of human umbilical cord mesenchymal stem cells in vitro to promote pancreatic gene expression. Mol Med Rep 8: 769-774, 2013.

11. Phermthai T, Odglun Y, Julavijitphong S, et al: A novel method to derive amniotic fluid stem cells for therapeutic purposes. BMC Cell Biol 11: 79, 2010
12. Troyer DL and Weiss ML: Wharton's jelly-derived cells are a primitive stromal cell population. StemCells 26: 591-599, 2008.

13. Kim J, Lee Y, Kim H, et al: Human amniotic fluid-derived stem cells have characteristics of multipotent stem cells. Cell Prolif 40: 75-90, 2007.

14. Schneider RK, Püllen A, Kramann R, et al: Long-term survival and characterisation of human umbilical cord-derived mesenchymal stem cells on dermal equivalents. Differentiation 79: 182-193, 2010

15. Atlasi Y, Mowla SJ, Ziaee SA and Bahrami AR: OCT-4, an embryonic stem cell marker, is highly expressed in bladder cancer. Int J Cancer 120: 1598-1602, 2007.

16. Hochedlinger K, Yamada Y, Beard C and Jaenisch R: Ectopic expression of Oct-4 blocks progenitor-cell differentiation and causes dysplasia in epithelial tissues. Cell 121: 465-477, 2005.

17. Mueller T, Luetzkendorf J, Nerger K, Schmoll HJ and Mueller LP: Analysis of OCT4 expression in an extended panel of human tumor cell lines from multiple entities and in human mesenchymal stem cells. Cell Mol Life Sci 66: 495-503, 2009.

18. Kaltz N, Funari A, Hippauf S, et al: In vivo osteoprogenitor potency of human stromal cells from different tissues does not correlate with expression of POU5F1 or its pseudogenes. Stem Cells 26: 2419-2424, 2008.

19. Lengner CJ, Camargo FD, Hochedlinger K, et al: Oct4 expression is not required for mouse somatic stem cell self-renewal. Cell Stem Cell 1: 403-415, 2007.

20. Carlin R, Davis D, Weiss M, Schultz B and Troyer D: Expression of early transcription factors Oct- 4 , Sox- 2 and Nanog by porcine umbilical cord (PUC) matrix cells. Reprod Biol Endocrinol 4: 8,2006

21. Can A and Karahuseyinoglu S: Concise review: human umbilical cord stroma with regard to the source of fetus-derived stem cells. Stem Cells 25: 2886-2895, 2007.

22. Weinberg MS, Barichievy S, Schaffer L, Han J and Morris KV: An RNA targeted to the HIV-1 LTR promoter modulates indiscriminate off-target gene activation. Nucleic Acids Res 35: 7303-7312, 2007.

23. Cockrell AS and Kafri T: Gene delivery by lentivirus vectors. Mol Biotechnol 36: 184-204, 2007.

24. Peng $X$, Zhang $X$ and Zeng B: Locally administered lentivirus-mediated siRNA inhibits wear debris-induced inflammation in murine air pouch model. Biotechnol Lett 30: 1923-1929, 2008

25. Miyoshi N, Ishii H, Nagai K, et al: Defined factors induce reprogramming of gastrointestinal cancer cells. Proc Natl Acad Sci USA 107: 40-45, 2010. 